\title{
BMJ Open Improving gender equity in critical care medicine: a protocol to establish priorities and strategies for implementation
}

\author{
Jeanna Parsons Leigh (1) , ${ }^{1,2}$ Chloe de Grood, ${ }^{2}$ Sofia Ahmed, ${ }^{3}$ Karen Bosma,, 4 \\ Karen E A Burns, ${ }^{6,7}$ Robert Fowler, ${ }^{7,8}$ Alison Fox-Robichaud (1) , ${ }^{9}$ Sangeeta Mehta, ${ }^{7}$ \\ Tina Mele (D) , ${ }^{4,10}$ Sharon E Straus, ${ }^{7,11}$ Nubia Zepeda, ${ }^{2}$ Laryssa Kemp, ${ }^{2}$ \\ Kirsten Fiest (D) , ${ }^{12,13}$ Henry Thomas Stelfox ${ }^{13,14}$
}

To cite: Parsons Leigh J, de Grood C, Ahmed S, et al. Improving gender equity in critical care medicine: a protocol to establish priorities and strategies for implementation. BMJ Open 2020;10:e037090. doi:10.1136/ bmjopen-2020-037090

- Prepublication history and additional material for this paper are available online. To view these files, please visit the journal online (http://dx.doi. org/10.1136/bmjopen-2020037090).

KF and HTS are joint senior authors.

Received 20 January 2020 Revised 09 April 2020 Accepted 19 May 2020
Check for updates

(C) Author(s) (or their employer(s)) 2020. Re-use permitted under CC BY-NC. No commercial re-use. See rights and permissions. Published by BMJ.

For numbered affiliations see end of article.

Correspondence to Dr Jeanna Parsons Leigh; j.parsonsleigh@dal.ca

\section{ABSTRACT}

Introduction While the number of women entering medical school now equals or surpasses the number of men, gender equity in medicine has not been achieved. Women continue to be under-represented in leadership roles (eg, deans, medical chairs) and senior faculty positions. In addition, women do not enter medical specialties as often as men, which can have important implications for work environment, reimbursement and the delivery of patient care. Compared with other medical specialties (eg, anaesthesiology, dermatology, etc), critical care medicine is a medical specialty with some of the lowest representation of women. While strategies to improve gender equity in critical care medicine exist in the published literature, efforts to comprehensively synthesise, prioritise and implement solutions have been limited. The objective of this programme of work is to establish priorities for the development and implementation of key strategies to improve the outcomes, well-being and experiences of women in critical care in Canada.

Methods and analysis Three phases encompass this programme of work. In phase I, we will catalogue published strategies focused on improving gender inequity across medical specialties through a scoping review. In phase II, we will conduct a modified Delphi consensus process with decision-makers, physicians and researchers to identify key strategies (identified in phase I and proposed by participants in phase II) for improving gender inequity in the specialty of critical care medicine. Finally, in phase III, we will conduct a 1-day stakeholder meeting that engages participants from phase II to build capacity for the development and implementation of top ranked strategies. Data analyses from this programme of work will be both quantitative and qualitative.

Ethics and dissemination The proposed programme of work is a foundational step towards establishing targeted strategies to improve gender inequity in the medical specialty of critical care medicine. Strategies will be prioritised by stakeholders, mapped to preidentified drivers of gender equity in the specialty and be scalable to institutional needs. A final report of our results including the list of top prioritised strategies and implementation objectives will be disseminated to panel participants,
Strengths and limitations of this study

- This programme of work is the first to prioritise strategies to address gender inequity in critical care medicine.

- Scoping review enables mapping of literature and catalogue of suggested strategies from published literature.

- The scoping review may not capture articles not published in the peer-reviewed literature (ie, grey literature).

- RAND/University of California Los Angeles (UCLA) appropriateness method is useful when coming to consensus on topics that have diverse opinions or no clear way forward.

- Strategies will be refined by critical care medicine stakeholders and therefore may not be broadly applicable across medical specialties.

critical care leadership teams and major critical care societies who are partners in this work, around the country to facilitate uptake at the local level.

The University of Calgary Conjoint Health Research Ethics Board has approved this study (REB16-0890).

\section{INTRODUCTION}

Throughout North America, women comprise at least half of medical school graduates, ${ }^{1-3}$ yet true gender equity (ie, equal status, pay, rights and opportunities regardless of gender) in medicine has not been achieved. ${ }^{4}$ Men continue to outnumber women with regard to leadership positions and tenure, ${ }^{56}$ overall compensation, ${ }^{7-9}$ as lead authors on publications or as journal editors, ${ }^{10}$ on clinical guideline committees, ${ }^{11}$ as speakers at medical conferences, ${ }^{12}{ }^{13}$ and as recipients of research funding. ${ }^{14} 15$ In the USA, only $20 \%$ of full-time faculty in departments of medicine are women. ${ }^{5}$ Compared with men, 
women are more likely to leave careers in academic medicine prematurely. ${ }^{1617}$ These disparities are further exacerbated in medical specialties. ${ }^{1}$ For example, less than $40 \%$ of specialists in Canada are women, and in a number of medical specialties (eg, general surgery, urology) this gap does not appear to be narrowing. ${ }^{18}$

Critical care medicine is a clinical medical specialty with some of the lowest representation of women. ${ }^{1}$ Research has demonstrated the existence of gender disparity in critical care medicine in both national and international settings. ${ }^{411} 131920$ Furthermore, a research initiative led by members of our team confirmed that only $20 \%$ of critical care faculty and $28 \%$ of critical care trainees at the 13 Canadian Universities with critical care training programmes were women. ${ }^{21}$ This study demonstrated both personal (eg, underestimated self competence) and professional (eg, difficulty advancing career) costs of gender inequity for women in critical care, and generated a framework of perceived drivers (ie, influencing factors), implications (ie, associated consequences) and strategies to attract and retain women in the specialty. ${ }^{21}$ To our knowledge, this was the first Canadian study using in-depth qualitative research methodologies to comprehensively explore and document key strategies to address gender inequity in a medical specialty. However, discussion, prioritisation and endorsement of key strategies to address gender inequities in critical care have not been comprehensively evaluated. Strategic and informed action is needed to address identified gender disparities in practice.

\section{Overarching objective of the current project}

To establish priorities for the development and implementation of key strategies to improve the representation, participation, well-being and experiences of women in critical care in Canada.

\section{Specific objectives}

1. To build on the strategies identified in our national qualitative study ${ }^{18}$ by conducting a scoping review of published strategies to address gender inequity across medical specialties.

2. To reach consensus on key strategies to reduce gender inequities in critical care.

3. To engage stakeholders in discussing these priorities and build capacity for the development and implementation of key strategies.

\section{METHODS AND ANALYSIS}

The proposed programme of work aims to identify key strategies to reduce gender inequities in critical care medicine and develop a stakeholder endorsed implementation plan through three phases of work. In phase I, through a scoping review, we will catalogue published strategies to address the gender gap in medical specialties. Phase II will involve a modified Delphi consensus process ${ }^{22}$ with decision-makers, physicians and researchers in critical care to identify key strategies to address gender inequities in the specialty. In phase III, we will conduct a 1-day stakeholder meeting that engages participants from phase II to build capacity for the development and implementation of key strategies. Data analyses will be both quantitative and qualitative in nature.

\section{Phase l: scoping review}

\section{Objective}

We will conduct a scoping review of published strategies (eg, mentorship programmes that span institutions, opportunities for modified role descriptions, etc) to address gender inequities in medical specialties.

The scoping review will be guided by the methods of Joanna Briggs Institute Methodology ${ }^{23}$ for Scoping Reviews and has been registered in Open Science Framework (https://osf.io/ek7yc/). Medical specialties include all those identified by the Canadian Medical Association. ${ }^{1}$ Strategies will be defined as any individual or multicomponent plan of action intervention, policy or solution that aims to address or mitigate gender inequity. Gender inequity refers to differences in the treatment, representation, opportunities and compensation between self-identified men and women. ${ }^{24}$

\section{Search strategy}

Medline, Embase, Scopus, Web of Science and Educational Resources Information Center (ERIC) will be searched for any relevant articles published in this area from their inception until November 2019. Reference lists of included articles will be searched for additional relevant articles. Further information will be requested from authors if needed. In preparation for this protocol manuscript, search terms were developed with an experienced information scientist around three categories: gender inequity, medical specialties and career progression including keywords and relevant antonyms and synonyms (online supplementary additional file 1 ).

\section{Study selection}

We will include citations that refer to strategies (interventions, actions or solutions) involving physicians (including faculty, attendings, fellows, residents and medical students) that address gender inequity. All original qualitative and quantitative research and systematic and non-systematic reviews, opinions and editorial citations will be considered for inclusion. Pilot testing of citation screening will occur ahead of title and abstract screening by two investigators and a random selection of 50 citations from the search will be pilot tested until screening is reliable (kappa statistic of $>0.8$ ). Screening will occur in two sequential steps: (1) title and abstract screening and (2) full-text screening. Both steps will be conducted by two investigators ( $\mathrm{CdG}, \mathrm{LK}$ ), independently, with discrepancies addressed through discussion. If necessary, a third reviewer (JPL) will reconcile outstanding differences in screening decisions. Citations will be screened in DistillerSR (Evidence Partners, Ottawa, Canada). 


\section{Data abstraction}

Data abstraction will occur independently in duplicate by two investigators (CdG, LK) in DistillerSR. Prior to starting data abstraction, we will pilot test the data extraction form using 10 randomly selected citations. We will extract relevant study characteristics (eg, year, country, study design), strategy to address gender inequity (eg, promote women in leadership) and medical specialty.

\section{Analysis}

An inductive qualitative approach will be used by two experienced qualitative investigators (JPL, CdG) to synthesise and catalogue a comprehensive list of key strategies (identified from the scoping review) to improve gender inequities by medical specialty. Analysis will draw from the modified thematic content analysis proposed by Braun and Clarke. ${ }^{21} 25$ We will conduct a narrative synthesis to summarise and explain the findings. Quantitative data will be summarised descriptively using numerical counts and percentages as appropriate with Stata statistical software (StataCorp). We will conduct qualitative analyses in NVivo (QSR International, Burlington, Massachusetts, USA).

\section{Outcome}

A catalogue of key strategies to address gender inequity in medical specialties and in-person stakeholder consultation to review the results.

\section{Phase II: modified Delphi consensus process Objective}

A modified Delphi process ${ }^{22}$ will be conducted with a diverse group of expert panellists (decision-makers/ opinion leaders, front-line clinicians (MD) and researchers) in critical care to review, rate and develop a comprehensive list of key strategies to address gender inequity in critical care medicine.

\section{Selection of panellists}

The panel will include a diverse group of stakeholders with an interest in addressing gender inequities in critical care. There is no predetermined number of participants for this type of work; however, it is important to have similar representation of participants across stakeholder groups. ${ }^{26} 27$ We will ensure gender parity (self-identified by participants) across the critical care participant list, including representation from three main groups: decision-makers or opinion leaders (eg, department heads, society leaders), physicians (staff and trainee) and researchers (eg, PhD scientists). The steering committee for this study, composed of 11 intensive care unit physicians, researchers and decision-makers, will purposively nominate up to 50 panellists to ensure diversity of representation across the specialty of critical care medicine. A minimum of 45 responses would be needed to achieve a $90 \%$ response rate. In order to achieve our target of 45-50 panellists, we will first invite 50 potential panellists and then invite new potential panellists every 2 weeks thereafter until we have reached our target. All potential panellists will be identified by the steering committee in advance of each round of invitations. Specifically, decisionmakers, physicians and researchers with different expertise, roles and affiliations from national and international organisations within Canada, the USA and the UK will be invited to a 1-day meeting to establish key priorities and plans for the development of key strategies to support gender equity in critical care. Representatives from these countries will be selected based on pre-existing relationships (with the steering committee) with the Canadian Critical Care Society (CCCS) https://canadiancriticalcare.org/, the Society of Critical Care Medicine https:// www.sccm.org/Home (SCCM) and the Intensive Care Society (UKICS) (http://members.ics.ac.uk) as well as researchers at international academic institutions. Representatives from CCCS, SCCM and UKICS will be invited in a decision-maker/opinion leader capacity.

\section{Consensus rating process}

A modified Delphi process ${ }^{22}$ will be conducted to iteratively review and revise candidate strategies for implementation in Canadian critical care. Panellists will participate in two electronic rounds of review using the distributed survey instrument. Panellists will be asked to rate which key strategies are essential for implementation to optimise gender equity in critical care. Open text fields will be presented after each strategy to enable respondents to provide comments on the strategy or suggest additional strategies for consideration. Successive rounds will only include strategies for which consensus was not achieved. Although we plan to conduct two rounds of review, we are prepared to conduct a third round (either remotely or in-person at the stakeholder meeting) if consensus is not achieved after the first two rounds.

\section{Rating instrument}

An electronic survey instrument, including all key strategies identified in phase I, will be developed using online survey software (Qualtrics Provo, Utah, USA). Unique strategies will be aggregated into categories and included in an importance-rating instrument developed using the Joint Commission's Attributes of Core Performance Measures and Associated Evaluation Criteria. ${ }^{28}$ Each category will have a definition, a list of key strategies and will be presented across a validated 9-point Likert scale (ranging from $1=$ non-essential to 9 =essential) for addressing gender inequity in critical care medicine in Canada. $^{22}$

\section{Data analysis}

We will summarise panellist ratings quantitatively using medians and IQRs. Consensus will be defined as strategies that receive a median rating of 1-3 or 7-9 and where fewer than two panellists rated the strategy outside the median tertile. Strategies will be classified as uncertain when the median rating is 4-6 or when two or more panellists rated the elements outside of the 1-3 or 7-9 tertiles. Disagreement will be defined as three or more panellists rating the 
strategy as 1-3 and at least three panellists rating the same strategy as $7-9$. With this approach, we will ensure that further rating and discussion occur when polarisation in ratings is identified. We will also conduct a secondary, stratified analysis by the demographic variables of sex and level of training.

\section{Outcome}

We aim to develop a list of key potential strategies to implement in critical care medicine departments across Canada to improve the representation, participation, well-being and experiences of women in critical care.

\section{Phase III: stakeholder forum \\ Approach}

We will hold a 1-day stakeholder meeting including decision-makers, physicians and researchers to establish priorities for the development and implementation of key strategies to support gender equity in critical care.

\section{Sampling and recruitment}

The overall goal of this meeting will be to develop and implement key strategies and shared initiatives to address gender inequities in critical care. To achieve this goal, we will engage the steering committee from phase II in an in-person event and assemble stakeholders by inviting those who participated in phase II.

\section{Meeting}

The meeting will be conducted by a facilitator with experience leading focus groups, panels and priority setting meetings (NZ), and will include a keynote address on the state of gender equity in medicine, presentation of background information (ie, synthesis of existing literature on the gender gap in critical care, including a framework of key strategies to improve gender equity), establishment of group priorities for improving gender equity (eg, small group discussions of feasibility and implementation), a prioritisation process (eg, small groups (8-10 participants per group) will discuss and prioritise key strategies to improve gender equity) and group deliberation (online supplementary additional file 2).

\section{Data analysis}

Following the meeting, support staff who took notes on deliberations and prioritisations will compare findings and resolve any differences through discussion with the investigators who attended the event. The audio recording of the meeting will be transcribed verbatim. Two investigators (CdG, LK) will independently analyse the transcriptions thematically according to standard principles of qualitative research drawing on grounded theory using a process of open, axial and selective coding. ${ }^{21}$ Qualitative analyses will also consider demographic variables (eg, sex, gender, level of training, etc) identified in phase II. The result will be a robust and comprehensive report describing needs and barriers to close the gender gap in critical care, decisions on shared priorities for the development and implementation of key strategies to reduce gender imbalance, opportunities for a future research agenda and the most relevant and feasible approaches and methods to address identified priorities. Meeting participants will have the opportunity to review and provide feedback on the report prior to broad dissemination.

\section{Outcome}

An implementation plan for key strategies to improve gender inequities in critical care that can also be tailored to academic institutions and professional bodies outside of critical care.

\section{Patient and public involvement}

Patients and members of the public will not be involved in this study.

\section{ETHICS AND DISSEMINATION Dissemination}

Gender inequity in medicine is a pressing moral, social and economic issue. ${ }^{2}{ }^{3}$ In critical care medicine, the gender gap has been linked to considerable adverse personal, professional and group level implications that adversely impact women. ${ }^{21}$ To attract and retain women in critical care, strategic action is needed to systematically address gender inequity. The proposed programme of work is a first step towards establishing international collaborations to develop and implement shared initiatives, targeted strategies and a future research agenda to reduce gender inequity in the specialty. Key strategies will be prioritised, mapped to preidentified drivers and implications of gender inequity in the field, and modifiable to institutional needs. A final report of our results including the list of top prioritised strategies and implementation objectives will be disseminated to panel participants, critical care leadership teams around the country to facilitate uptake at the local level. We will also share our findings with major critical care societies who are partners in this work. Although prioritised strategies will be developed for critical care medicine in Canada, we anticipate that there will be broad applicability across medical specialties given the diversity of strategies compiled in phase I (ie, not limited to the critical care literature) and the inclusion of stakeholders from diverse geographic (eg, 3 countries, 10 provinces) and training (eg, surgery, anaesthesia, internal medicine, etc) backgrounds. We anticipate strategies will be targeted to a diverse group of settings which will be dependent on group prioritisation (phase II) and discussion (phase III). The University of Calgary Conjoint Health Research Ethics Board has approved this study (REB16-0890).

\footnotetext{
Author affiliations

${ }^{1}$ School of Health Administration, Dalhousie University, Halifax, Nova Scotia, Canada ${ }^{2}$ Department of Critical Care Medicine, University of Calgary, Calgary, Alberta, Canada

${ }^{3}$ Department of Medicine, University of Calgary, Calgary, Alberta, Canada

${ }^{4}$ Division of Critical Care Medicine, London Health Sciences Centre, London, Ontario, Canada
} 
${ }^{5}$ Department of Medicine, Western University Schulich School of Medicine and Dentistry, London, Ontario, Canada

${ }^{6}$ Critical Care, St. Michael's Hospital, Toronto, Ontario, Canada

${ }^{7}$ Interdepartmental Division of Critical Care Medicine, Department of Medicine, University of Toronto, Toronto, Ontario, Canada

${ }^{8}$ Sunnybrook Research Institute, Toronto, Ontario, Canada

${ }^{9}$ Department of Medicine, Division of Critical Care Medicine, McMaster University, Hamilton, Ontario, Canada

${ }^{10}$ Department of Medicine, Western University, London, Ontario, Canada

${ }^{11} \mathrm{Li}$ Ka Shing Knowledge Institute, St. Michael's Hospital, Toronto, Ontario, Canada

${ }^{12}$ Departments of Critical Care Medicine, Department of Psychiatry \& Hotchkiss Brain Institute and Department of Community Health Sciences, University of Calgary Cumming School of Medicine, Calgary, Alberta, Canada

${ }^{13}$ O'Brien Institute for Public Health, Calgary, Alberta, Canada

${ }^{14}$ Department of Critical Care Medicine, Department of Community Health Sciences, University of Calgary, Calgary, Alberta, Canada

Twitter Alison Fox-Robichaud @drfoxrob

Acknowledgements Helen Lee Robertson and Lorraine Toews (Research Librarians, University of Calgary).

Contributors JPL, HTS, KF, SA, KB, KEAB, RF, AF-R, SM, TM and SES contributed to the conception of this study protocol. JPL, HTS, KF, CdG, LK and NZ wrote the initial draft of this protocol and SA, KB, KEAB, RF, AF-R, SM, TM and SES iteratively reviewed and refined the protocol manuscript. All authors will be accountable for al aspects proposed in this protocol and contribute to the acquisition and analysis of data described in this protocol.

Funding This study is supported by a CIHR Planning and Dissemination GrantInstitute Community Support.

Competing interests None declared.

Patient and public involvement Patients and/or the public were not involved in the design, or conduct, or reporting, or dissemination plans of this research.

Patient consent for publication Not required.

Provenance and peer review Not commissioned; externally peer reviewed.

Open access This is an open access article distributed in accordance with the Creative Commons Attribution Non Commercial (CC BY-NC 4.0) license, which permits others to distribute, remix, adapt, build upon this work non-commercially, and license their derivative works on different terms, provided the original work is properly cited, appropriate credit is given, any changes made indicated, and the use is non-commercial. See: http://creativecommons.org/licenses/by-nc/4.0/.

ORCID iDs

Jeanna Parsons Leigh http://orcid.org/0000-0002-8408-674X

Alison Fox-Robichaud http://orcid.org/0000-0001-9912-3606

Tina Mele http://orcid.org/0000-0002-6617-9790

Kirsten Fiest http://orcid.org/0000-0002-7299-6594

\section{REFERENCES}

1 Canadian Medical Association. Number and percent distribution of physicians by specialty and sex, Canada 2018, 2018. Available: https://www.cma.ca/Assets/assets-library/document/en/advocacy/ 06-spec-sex.pdf

2 Cabot RC. Women in medicine. 1915. JAMA 2015;314:1076.

3 Morgan AU, Chaiyachati KH, Weissman GE, et al. Eliminating genderbased bias in academic medicine: more than naming the "elephant in the room". J Gen Intern Med 2018;33:966-8.

4 Sreedharan R, Perez-Protto S. Women in critical care medicine. Society of critical care medicine, 2017. Available: http://www.sccm. org/Communications/Critical-Connections/Archives/Pages/Womenin-Critical-Care-Medicine.aspx
5 Carr PL, Gunn CM, Kaplan SA, et al. Inadequate progress for women in academic medicine: findings from the National faculty study. $J$ Womens Health 2015;24:190-9.

6 Metaxa V. Is this (still) a man's world? Crit Care 2013:17:112.

7 Jena AB, Olenski AR, Blumenthal DM. Sex differences in physician salary in US public medical schools. JAMA Intern Med 2016;176:1294-304.

8 Appleby J. Is there equal pay in healthcare? not if you are a doctor. BMJ 2012;345:e6191.

9 Rimmer A. Review of $£ 10000$ gender pay gap in medicine is launched. BMJ 2018;361:k2366.:k2366.

10 Filardo G, da Graca B, Sass DM, et al. Trends and comparison of female first authorship in high impact medical journals: observational study (1994-2014). BMJ 2016;352:i847.

11 Mehta S, Burns KEA, Machado FR, et al. Gender parity in critical care medicine. Am J Respir Crit Care Med 2017;196:425-9.

12 Fishman M, Williams WA, Goodman DM, et al. Gender differences in the authorship of original research in pediatric journals, 2001-2016. J Pediatr 2017;191:244-9.

13 Mehta S, Rose L, Cook D, et al. The SPEAKER gender gap at critical care conferences. Crit Care Med 2018;46:991-6.

14 Witteman $\mathrm{HO}$, Hendricks M, Straus S, et al. Are gender gaps due to evaluations of the applicant or the science? a natural experiment at a national funding agency. Lancet 2019;393:531-40.

15 Burns KEA, Straus SE, Liu K, et al. Gender differences in grant and personnel Award funding rates at the Canadian Institutes of health research based on research content area: a retrospective analysis. PLoS Med 2019;16:e1002935.

16 Buddeberg-Fischer B, Stamm M, Buddeberg C, et al. The impact of gender and parenthood on physicians' careers--professional and personal situation seven years after graduation. BMC Health Serv Res 2010;10:40.

17 Carr PL, Ash AS, Friedman $\mathrm{RH}$, et al. Relation of family responsibilities and gender to the productivity and career satisfaction of medical faculty. Ann Intern Med 1998;129:532.

18 Mascarenhas A, Moore JE, Tricco AC, et al. Perceptions and experiences of a gender gap at a Canadian research Institute and potential strategies to mitigate this gap: a sequential mixed-methods study. CMAJ Open 2017;5:E144-51.

19 Modra L, Yong S, Austin D. Women in leadership in intensive care medicine. ICU Manag Pract 2016;16.

20 Burns KEA, Fox-Robichaud A, Lorens E, et al. Gender differences in career satisfaction, moral distress, and incivility: a national, crosssectional survey of Canadian critical care physicians. Can J Anaesth 2019;66:503-11.

21 Leigh JP, Grood Cde, Ahmed SB, et al. Toward gender equity in critical care medicine: a qualitative study of perceived drivers, implications, and strategies. Crit Care Med 2019;47:E286-91.

22 Fitch K, Bernstein SJ, Mcdonnell J, et al. The RAND / UCLA appropriateness method user 's manual, 2001. Available: https:// www.rand.org/content/dam/rand/pubs/monograph_reports/2011/ MR1269.pdf [Accessed 19 Nov 2019].

23 The Joanna Briggs Institute. The joanna briggs institute reviewers' manual 2015, 2015: 1-24.

24 World Health Organization. Gender, 2019. Available: https://www. who.int/gender-equity-rights/understanding/gender-definition/en/ [Accessed 19 Nov 2019].

25 Braun V, Clarke V. Using thematic analysis in psychology. Qual Res Psychol 2006;3:77-101.

26 McMillan SS, King M, Tully MP. How to use the nominal group and Delphi techniques. Int J Clin Pharm 2016;38:655-62.

27 Akins RB, Tolson H, Cole BR. Stability of response characteristics of a Delphi panel: application of bootstrap data expansion. BMC Med Res Methodol 2005;5:37.

28 The Joint Commission. Attributes of core performance measures and associated evaluation criteria, 2012. Available: https://www. jointcommission.org/assets/1/18/Attributes_of_Core_Performance_ Measures_and_Associated_Evaluation_Criteria.pdf [Äccessed 19 Nov 2019]. 